\title{
Les défis du diagnostic de l'infection à chikungunya : Présentation d'un tableau clinique atypique
}

\author{
Craig J', Klowak $\mathrm{M}^{2}$, Boggild $\mathrm{AK}^{1,3,4^{*}}$ \\ ${ }^{1}$ Division des maladies infectieuses, Département de médecine, Université de Toronto, Toronto (Ontario) \\ ${ }^{2}$ Faculté des sciences de la vie, Université McMaster, Toronto (Ontario) \\ ${ }^{3}$ Unité des maladies tropicales, Réseau universitaire de santé, Hôpital général de Toronto, Toronto (Ontario) \\ ${ }^{4}$ Laboratoires de Santé publique Ontario, Santé publique Ontario, Toronto (Ontario) \\ *Auteure-ressource : andrea.boggild@utoronto.ca
}

\section{Résumé}

Le chikungunya est devenu une préoccupation de santé publique importante, une situation attribuable en partie à l'augmentation des voyages internationaux. Les récentes éclosions observées dans les pays de la Caraïbe et le signalement d'un premier cas d'infection autochtone aux États-Unis, donnent à craindre une possible augmentation du nombre de cas au Canada. Le chikungunya ayant un grand nombre de symptômes cliniques en commun avec d'autres maladies transmises par les arthropodes, telles que la dengue, le diagnostic clinique de la maladie est compliqué. Dans cet article, nous présentons un tableau clinique atypique de chikungunya observé chez un homme au retour d'un voyage à Haïti. Le diagnostic microbiologique, le traitement, le pronostic et les implications pour la santé publique aideront à préparer les cliniciens à la prise en charge de cet agent pathogène émergent.

\section{Introduction}

Le chikungunya est une maladie virale transmise par les moustiques, dont le virus est de plus en plus reconnu dans le monde entier comme étant un agent pathogène émergent. En décembre 2013, une transmission locale du virus du chikungunya a été signalée pour la première fois dans les Amériques, ce qui a mené l'Agence de la santé publique du Canada (ASPC) à aviser les cliniciens et le personnel de la santé publique d'envisager la présence possible du virus chez les voyageurs de retour au pays présentant une fièvre et une polyarthralgie (1). Un récent rapport des Centers for Disease Control and Prevention (CDC) des États-Unis identifie 25 pays de la Caraïbe où des cas d'infection autochtone à chikungunya ont été signalés (2). Dans le présent article, nous présentons un cas atypique d'infection à chikungunya observé chez un voyageur de retour de Haïti, dans le but de mettre en lumière les caractéristiques cliniques que le chikungunya a en commun avec d'autres infections à arbovirus, telles que la dengue, ainsi que les outils diagnostiques importants dont disposent les cliniciens, et de répondre aux préoccupations soulevées par la propagation à l'échelle mondiale de l'infection.

\section{Contexte}

L'infection à chikungunya est causée par un arbovirus de la famille des Togaviridae et est transmise essentiellement par les moustiques Aedes aegypti et dans une moindre mesure par ceux de l'espèce Aedes albopictus (3).

Le virus du chikungunya a été décrit pour la première fois en Tanzanie en 1953 (4) et est considéré endémique dans certaines régions de l'Afrique occidentale. En septembre 2014, on portait à 88 le nombre de pays ayant signalé la transmission de cas d'infection par ce virus, ce qui comprenait les pays d'Afrique, d'Europe, d'Océanie, d'Asie et, plus récemment, les pays des Amériques (5). Le premier cas d'infection autochtone à chikungunya observé en zone tempérée a été recensé en Italie en 2007, le cas index suspecté étant une personne revenant d'Inde (6). Le vecteur suspecté, Aedes albopictus, aurait acquis une mutation génétique sous l'effet de la pression écologique qui lui aurait permis de supplanter Aedes aegypti en tant que principal vecteur (3). Le moustique Aedes albopictus étant largement répandu dans le sud-est des États-Unis, la transmission locale de l'infection fait l'objet de préoccupations croissantes dans ces États. En fait, le premier cas d'infection à chikungunya contractée 
localement aux États-Unis a été signalé chez un homme de Floride (7). La transmission de l'infection ne devrait pas se produire au Canada, étant donné que l'on ne trouve pas de moustiques Aedes sous ce type de climat (1).

Dans la plupart des cas, l'infection se manifeste par une polyarthralgie symétrique invalidante accompagnée de fièvre (8). Les articulations couramment atteintes comprennent celles des chevilles, des genoux, les articulations métacarpophalangiennes et métatarsiennes, celles des épaules, des coudes et des poignets. Chez près d'un tiers des patients, on peut observer un gonflement des articulations, mais l'épanchement articulaire est rare (8). Après une période d'un à trois jours, il apparaît souvent une éruption cutanée maculopapulaire diffuse, qui épargne généralement le visage. Les douleurs articulaires disparaissent en général au bout de plusieurs semaines; dans de nombreux cas, toutefois, elles peuvent persister pendant des mois, voire des années, souvent en ayant des répercussions importantes sur la qualité de vie du patient (9).

Le diagnostic clinique est compliqué car le chikungunya a des signes et symptômes communs avec d'autres maladies, telles que l'infection à parvovirus B19 et la dengue. La confirmation microbiologique est nécessaire et repose habituellement sur la détection de la présence d'anticorps IgM ou IgM dans le sérum au moyen d'un essai immuno-enzymatique (ELISA). Les anticorps IgM sont souvent détectés deux à six jours après l'apparition des symptômes alors que les anticorps IgG apparaissent généralement durant la phase de convalescence de la maladie et peuvent être présents pendant des années (10). L'analyse d'échantillons sériques, plasmatiques ou de liquide céphalorachidien (LCR) selon une technique de transcription inverse suivie d'une réaction en chaîne de la polymérase (RT-PCR) est la méthode d'analyse la plus sensible; elle est offerte à des fins de recherche par le Laboratoire national de microbiologie de Winnipeg, au Manitoba (11). Le cas que nous présentons met en lumière certaines des incertitudes qui caractérisent le diagnostic du chikungunya.

Le traitement du chikungunya est généralement un traitement de soutien qui repose sur la prescription d'agents anti-inflammatoires non stéroïdiens, la consommation de liquides et le repos. Les corticostéroïdes sont réservés aux symptômes arthritiques invalidants se manifestant au tout début de la phase aiguë de l'infection (3). Des recherches sont en cours sur l'utilisation potentielle d'anticorps monoclonaux, d'agents antiviraux et de vaccins $(12,13)$.

\section{Présentation du cas}

Le lendemain de son retour d'un séjour de 11 jours à Haïti, un homme âgé de 74 ans se présente aux urgences en se plaignant de constipation, de douleur abdominale et de l'apparition récente d'une éruption cutanée maculopapulaire diffuse sans desquamation touchant le thorax, le dos, les bras et les jambes. L'éruption cutanée n'est ni douloureuse ni prurigineuse. Un tomodensitogramme de l'abdomen révèle une diverticulite intéressant l'intestin grêle accompagnée d'une perforation possible au sein des tissus adipeux environnants. II est admis à l'hôpital pour y recevoir des soins de soutien, notamment par l'administration d'antibiotiques. La perforation est présumée avoir été consécutive à une diverticulose de l'intestin grêle compliquée par une constipation importante, révélée par un diagnostic antérieur.

Pendant son séjour à Haïti, il a travaillé comme travailleur de l'aide dans une clinique médicale locale. Avant de partir pour Haïti, il avait reçu une série complète de vaccins contre l'hépatite $A$ et $B$ et son médecin lui avait prescrit un traitement prophylactique antipaludéen à base de chloroquine, qu'il avait suivi rigoureusement. Le neuvième jour de son séjour, il se réveille en souffrant d'une douleur articulaire diffuse intense touchant les grosses et les petites articulations de ses membres supérieurs et inférieurs, accompagnée de frissons et d'une fièvre subjective. Pas de problèmes respiratoires ni gastro-intestinaux. Au bout de 48 heures, alors que ses douleurs articulaires se sont grandement estompées, apparaît une éruption cutanée tronculaire, accompagnée d'une constipation importante, qui le pousse à se rendre aux urgences.

Selon l'examen physique effectué au service des urgences, l'abdomen du patient n'est ni dur ni douloureux. Aucun gonflement articulaire n'est observé; toutefois, une éruption cutanée maculopapulaire recouvre le thorax et les membres supérieurs et inférieurs. Les examens cardiaque, respiratoire et neurologique ne révèlent rien d'anormal. Les analyses de laboratoire courantes sont effectuées (Tableau 1). Elles révèlent la présence d'une lymphocytopénie et d'une thrombocytopénie marquées; la radiographie thoracique effectuée lors de l'admission du patient, est normale. 
Tableau 1 : Analyses de laboratoire courantes effectuées lors de l'admission à l'hôpital ${ }^{1}$

\begin{tabular}{|c|c|c|}
\hline Investigation & Valeur & Plage de référence \\
\hline Hémoglobine & $143 \mathrm{~g} / \mathrm{L}$ & $132-170 \mathrm{~g} / \mathrm{L}$ \\
\hline Leucocytes & $9,9 \times 10^{9} / \mathrm{L}$ & $3,5-10 \times 10^{9} / \mathrm{L}$ \\
\hline - Neutrophiles & $8,6 \times 10^{9} / L$ & $2,5-7,5 \times 10^{9} / \mathrm{L}$ \\
\hline - Lymphocytes & $0,5 \times 10^{9} / L$ & $1,0-4,0 \times 10^{9} / L$ \\
\hline Plaquettes & $108 \times 10^{9} / \mathrm{L}$ & $130-400 \times 10^{9} / L$ \\
\hline SGOT & $36 \mathrm{U} / \mathrm{L}$ & $13-37 \mathrm{U} / \mathrm{L}$ \\
\hline GPT & $18 \mathrm{U} / \mathrm{L}$ & $10-40 \mathrm{U} / \mathrm{L}$ \\
\hline PhoA & $94 \mathrm{U} / \mathrm{L}$ & $40-120 \mathrm{U} / \mathrm{L}$ \\
\hline Bilirubine (totale) & $11 \mathrm{mmol} / \mathrm{L}$ & $3,0-20 \mathrm{mmol} / \mathrm{L}$ \\
\hline Sodium & $136 \mathrm{mmol} / \mathrm{L}$ & $135-145 \mathrm{mmol} / \mathrm{L}$ \\
\hline Potassium & $4 \mathrm{mmol} / \mathrm{L}$ & $3,5-5,0 \mathrm{mmol} / \mathrm{L}$ \\
\hline Bicarbonate & $22 \mathrm{mmol} / \mathrm{L}$ & $20-30 \mathrm{mmol} / \mathrm{L}$ \\
\hline Créatinine & $79 \mathrm{mmol} / \mathrm{L}$ & $55-105 \mathrm{mmol} / \mathrm{L}$ \\
\hline Lactate & $1,0 \mathrm{mmol} / \mathrm{L}$ & $0,5-2,0 \mathrm{mmol} / \mathrm{L}$ \\
\hline Uroculture & Négative & S.O. ${ }^{2}$ \\
\hline Hémoculture & Négative & S.O. ${ }^{2}$ \\
\hline $\begin{array}{l}\text { Test de détection rapide des } \\
\text { antigènes du paludisme }\end{array}$ & Négatif & S.O. ${ }^{2}$ \\
\hline
\end{tabular}

À l'hôpital, le patient reçoit des soins de soutien, dont l'administration intraveineuse d'une solution cristalloïde, et un traitement aux antibiotiques. II quitte l'hôpital pour être orienté en urgence vers une clinique spécialisée dans les maladies tropicales en vue de l'évaluation de sa présumée maladie liée aux voyages. La recherche d'anticorps IgG et IgM dirigés contre le virus de la dengue par la technique ELISA se révèle négative. L'analyse des selles à la recherche de Salmonella spp, Escherichia coli O157:H7, Campylobacter spp. et Shigella spp. se révèle négative. Le test ELISA révèle la présence d'anticorps IgM anti-chikungunya ce qui supporte un diagnostic probable d'une infection aiguë au virus du chikungunya. Sans un test de confirmation, tel un test de séroneutralisation, la possibilité de réactivité croisée avec d'autres alphavirus ne peut être exclue de manière définitive. Sa douleur abdominale disparaît à l'hôpital uniquement avec des soins de soutien, alors que l'arthrite et l'éruption cutanée mettent deux semaines à disparaître complètement.

\section{Discussion}

Nous présentons le tableau clinique d'un cas atypique d'infection aiguë à chikungunya observé chez un homme ayant effectué un séjour à Haïti, une région connue pour être aux prises avec une transmission intense et persistante de la dengue et du chikungunya. Bien que le patient présente initialement les symptômes classiques de polyarthrite symétrique suivis d'une éruption cutanée maculopapulaire, son tableau clinique est compliqué par la présence d'une douleur abdominale intense, d'une constipation et d'une thrombocytopénie, manifestations atypiques de l'infection à chikungunya. La nette amélioration observée en l'espace de 48 heures, est également inhabituelle étant donné que les douleurs articulaires ayant des répercussions sur la mobilité et la dextérité durent souvent des semaines, voire des mois (3). Bien que rares, d'autres manifestations atypiques de l'infection à chikungunya ont été publiées, notamment des manifestations neurologiques (dont encéphalite, convulsions et syndrome de Guillain-Barré), des manifestations cardiovasculaires (dont myocardites, insuffisance cardiaque et cardiopathie ischémique), des manifestations rénales (dont insuffisance rénale aiguë), des manifestations oculaires (dont névrite optique) ainsi que des manifestations atypiques d'éruption cutanée, d'ulcération et de phlyctène (14).

Le diagnostic différentiel de la fièvre et de la polyarthrite sans épanchement est vaste. Les causes courantes d'origine bactérienne comprennent la maladie de Lyme et l'endocardite infectieuse. Les causes fréquentes d'origine virale comprennent le parvovirus B19, l'hépatite $B$ et $C$, la rubéole, la dengue et d'autres arbovirus, notamment les virus de Mayaro, d'O'nyong-nyong, de Ross River, de la forêt de Barmah, de Sindbis et de la forêt de Semliki. Les causes non infectieuses comprennent les spondyloarthropathies séronégatives, l'arthrite 
rhumatoïde, les arthropathies induites par des dépôts de cristaux et l'arthrite post-infectieuse (réactive). À la lumière du risque épidémiologique et du tableau clinique caractérisant notre patient, les causes infectieuses les plus envisageables comprenaient la dengue et l'infection à chikungunya et dans une moindre mesure le parvovirus B19. Une comparaison des caractéristiques, du tableau clinique et des paramètres biologiques du chikungunya et de la dengue apparaît au Tableau 2. Les causes d'origine non infectieuses ont été jugées improbables en raison de la fièvre initiale du patient, de son éruption cutanée et de l'amélioration rapide des symptômes.

Tableau 2 : Tableau clinique et paramètres biologiques du chikungunya et de la dengue

\begin{tabular}{|c|c|c|}
\hline $\begin{array}{c}\text { Tableau clinique et paramètres } \\
\text { biologiques }\end{array}$ & Chikungunya & Dengue \\
\hline \multicolumn{3}{|l|}{ Caractéristiques de la maladie (19) } \\
\hline Période d'incubation & $\begin{array}{l}3-7 \text { jours } \\
\text { (plage } 2-12 \text { ) }\end{array}$ & $\begin{array}{l}4-7 \text { jours } \\
\text { (plage } 3-14 \text { ) }\end{array}$ \\
\hline $\begin{array}{l}\text { Rapport asymptomatique/ } \\
\text { symptomatique }\end{array}$ & $0,03 / 1-0,25 / 1$ & $2 / 1-10 / 1$ \\
\hline \multicolumn{3}{|l|}{ Tableau clinique $(3,8,9,17,19,20,21)$} \\
\hline Fièvre & Courante & Courante \\
\hline Arthralgie & Courante & Possible \\
\hline Polyarthrite (sans épanchement) & Courante & Non probable \\
\hline Myalgie & Possible & Courante \\
\hline Éruption cutanée & $\begin{array}{l}\text { Courante, souvent au } \\
\text { bout de } 1 \text { à } 4 \text { jours }\end{array}$ & $\begin{array}{l}\text { Courante, souvent au } \\
\text { bout de } 3 \text { à } 7 \text { jours }\end{array}$ \\
\hline Douleur abdominale & Non probable & Possible \\
\hline Douleur rétro-orbitale & Non probable & Courante \\
\hline Douleur articulaire chronique & $\begin{array}{l}\text { Courante, peut durer } \\
>2 \text { ans }\end{array}$ & Non probable \\
\hline Fatigue chronique & $\begin{array}{l}\text { Courante, peut durer } \\
>2 \text { ans }\end{array}$ & $\begin{array}{l}\text { Courante, peut durer } \\
\text { jusqu'à } 3 \text { mois }\end{array}$ \\
\hline \multicolumn{3}{|l|}{ Paramètres biologiques } \\
\hline Neutropénie & Possible & Courante \\
\hline Lymphopénie & Courante & Courante \\
\hline Thrombocytopénie & Possible & Courante \\
\hline
\end{tabular}

L'amélioration rapide des symptômes en l'absence de soins de soutien est atypique en présence d'une infection à chikungunya. Bien que s'agissant probablement de cas d'infection au tableau clinique atypique, des études in vitro (15) ont donné à penser que la chloroquine permettrait d'atténuer les symptômes de l'infection à chikungunya. Cet effet thérapeutique n'a toutefois pas été confirmé par des essais contrôlés à répartition aléatoire chez les humains (16); le rôle joué par la prophylaxie antipaludéenne à base de chloroquine dans l'atténuation des symptômes de notre patient reste donc incertain.

L'existence d'une constipation causant une douleur abdominale n'est pas non plus typique de l'infection à chikungunya. Dans une étude comparative menée en Inde, la douleur abdominale n'a été signalée par aucun des $131(0 \%)$ patients présentant une infection à chikungunya en phase aiguë alors qu'elle a été signalée par 22 des 104 patients (21\%) présentant une dengue en phase aiguë (17). Par contre, lors une éclosion survenue dans l'île de La Réunion, 47 \% des patients ont signalé des symptômes gastro-intestinaux, le nombre de cas de douleur abdominale ou de constipation n'ayant toutefois pas été indiqué (8). Étant donné l'évolution de la maladie, la constipation semble être associée à l'infection aiguë à chikungunya chez ce patient; toutefois, nous admettons la possibilité de l'existence de deux maladies sous-jacentes qui auraient contribué à ces symptômes. La présence concomitante d'une entérite à Salmonella spp. pourrait expliquer la constipation et la douleur abdominale, cette infection, endémique en Haïti, étant souvent associée à une constipation. Les coprocultures, effectuées sur des échantillons prélevés après l'administration d'antibiotiques, ce qui diminue considérablement la présence éventuelle de germes pathogènes, ont pu donner lieu à de faux négatifs.

\section{Conclusion}


Vu le nombre croissant de pays signalant de nouveaux cas de transmission du chikungunya, l'infection est en passe de devenir une préoccupation à l'échelle mondiale. La capacité de mutation du virus sous l'effet de la pression de sélection et l'augmentation des voyages internationaux donnent au chikungunya un potentiel épidémique important. Étant donné le tableau clinique diversifié de l'infection, les cliniciens doivent faire preuve de vigilance et envisager la présence d'une infection à chikungunya chez les patients présentant une fièvre et une polyarthralgie, quels que soient les autres signes cliniques et paramètres biologiques, à leur retour d'un pays à haut risque. Bien que le traitement repose généralement sur des soins de soutien, les symptômes des patients, notamment l'arthrite invalidante, peuvent persister plusieurs années, ce qui souligne l'importance que revêt la sensibilisation du public aux techniques appropriées de prévention des piqûres de moustiques en voyage, notamment l'utilisation de DEET ou d'un insectifuge à base d'icaridine et de vêtements protecteurs (18).

\section{Conflit d'intérêts}

Aucun

\section{Financement}

Aucun

\section{Références}

(1) Deilgat, M., Geduld, J., Drebot, M. Éclosion de chikungunya dans les Caraïbes (2013-2014). Relevé des maladies transmissibles au Canada, janvier 2014; 40(2):7-12. http://www.phac-aspc.gc.ca/publicat/ccdr-rmtc/14vol40/dr-rm4002/dr-rm40-02-chik-fra.php

(2) Centers for Disease Control and Prevention. Chikungunya in the Caribbean. wwwnc.cdc.gov/travel/notices/watch/chikungunya-caribbean

(3) Thiboutot MM, Kannan S, Kawalekar OU, Shedlock DJ, Khan AS, Sarangan G, et al. Chikungunya: A potentially emerging epidemic? PLoS Negl Trop Dis. 2010; 4:e623.

(4) Lumsden WH. An epidemic of virus disease in Southern Province, Tanganyika Territory, in 1952-53. II. General description and epidemiology. Trans R Soc Trop Med Hyg. 1955 Jan; 49(1):33-57.

(5) Centers for Disease Control and Prevention. Geographic distribution-Where has chikungunya virus been found? www.cdc.gov/chikungunya/geo/index.html

(6) Rezza G, Nicoletti L, Angelini R, Romi R, Finarelli AC, Panning M. Infection with chikungunya virus in Italy: An outbreak in a temperate region. Lancet. 2007 Dec 1; 370(9602):1840-6.

(7) McCarthy M. First case of locally acquired chikungunya is reported in US. BMJ. 2014 Jul $18 ; 349$.

(8) Borgherini G, Poubeau P, Staikowsky F, Lory M, Le Moullec N, Becquart JP, et al. Outbreak of chikungunya on Reunion Island: early clinical and laboratory features in 157 adult patients. Clin Infect Dis. 2007; 44:1401-7.

(9) Marimoutou C, Vivier E, Oliver M, Boutin JP, Simon F. Morbidity and impaired quality of life 30 months after chikungunya infection: Comparative cohort of infected and uninfected French military policemen in Reunion Island. Medicine (Baltimore). $2012 \mathrm{Jul} ;$ 91(4):212-9.

(10) Niedrig M, Zeller H, Schuffenecker I, Drosten C, Emmerich P, Rumer L, et al. International diagnostic accuracy study for the serological detection of chikungunya virus infection. Clin Microbiol Infect. 2009; 15:880-4.

(11) Schwartz KL, Giga A, Boggild AK. Chikungunya fever in Canada: Fever and polyarthritis in a returned traveller. CMAJ. 2014 Jul 8; 186(10):772-4.

(12) Chang LJ, Dowd KA, Mendoza FH, Saunders JG, Sitar S, Plummer SH. Safety and tolerability of chikungunya virus-like particle vaccine in healthy adults: A phase 1 dose-escalation trial. Lancet. 2014 Dec 6; 384(9959):2046-52. http://www.thelancet.com/journals/lancet/article/PIIS0140-6736(14)61185-5/fulltext

(13) Powers AM. Chikungunya virus control: Is a vaccine on the horizon? Lancet. 2014 Dec 6; 384(9959):2008-9. http://www.thelancet.com/journals/lancet/article/PIIS0140-6736(14)61290-3/fulltext

(14) Rajapakse S, Rodrigo C, Rajapakse A. Atypical manifestations of chikungunya infection. Trans R Soc Trop Med Hyg. 2010 Feb; 104(2):89-96.

(15) Khan M, Santhosh SR, Tiwari M, Lakshmana Rao PV, Parida M. Assessment of in vitro prophylactic and therapeutic efficacy of chloroquine against Chikungunya virus in vero cells. J Med Virol. 2010 May; 82(5):817-24.

(16) Chopra A, Saluja M, Venugopalan A. Effectiveness of chloroquine and inflammatory cytokine response in patients with early persistent musculoskeletal pain and arthritis following chikungunya virus infection. Arthritis Rheumatol. 2014 Feb; 66(2):319-26.

(17) Taraphdar D, Sarkar A, Mukhopadhyay BB, Chatterjee S. A comparative study of clinical features between monotypic and dual infection cases with Chikungunya virus and dengue virus in West Bengal, India. Am J Trop Med Hyg. 2012 Apr; 86(4):720-3. 
(18) Schofield, S. et Plourde, P. pour le Comité consultatif de la médecine tropicale et de la médecine des voyages (CCMTMV). Déclaration relative aux mesures de protection individuelle pour prévenir les piqûres ou morsures d'arthropodes. Relevé des maladies transmissibles au Canada 2012; 38(DCC-3):1-18.

(19) Chen LH, Wilson ME. Dengue and chikungunya in travelers. Curr Opin Infect Dis. 2010 Oct;23(5):438-44

(20) Queyriaux B, Simon F, Grandadam M, Michel R, Tolou H, Boutin JP. Clinical burden of chikungunya virus infection. Lancet Infect Dis. 2008 Jan;8(1):2-3.

(21) Potts JA, Rothman AL. Clinical and laboratory features that distinguish dengue from other febrile illnesses in endemic populations. Trop Med Int Health. $2008 \mathrm{Nov}$;13(11):1328-40. 